\title{
Affective Impressions of Various Disabilities Using the Semantic Differential Method
}

\author{
Junichi Takahashi \\ Faculty of Human Development and Culture \\ Fukushima University \\ Japan
}

\begin{abstract}
The present study measured affective impressions of disabilities (intellectual disability, physically handicapped, weakness, blindness, deafness, and developmental disability). The present study adopted the SD (semantic differential) method. Fifty participants rated each disability-related term on 20 adjective pairs presented as sixpoint scales. A factor analysis extracted three factors: Activity, Evaluation, and Potency. On the basis of scores for these factors, impressions of physically handicapped, weakness, blindness, and deafness were more negative than those of intellectual disability and developmental disability on the Activity factor. Participants negatively rated intellectual disability, physically handicapped, weakness, and developmental disability compared with blindness and deafness on the Evaluation factor. On the Potency factor, the participants negatively rated physically handicapped compared with other disabilities. The present results provide new evidence that affective impressions toward disabilities vary depending on the type of disability and on factors extracted by the SD method, considering the sensory/perceptual analysis underlying each factor.
\end{abstract}

Keywords: Affective impressions, Attitudes, Perception, Semantic differential (SD) method, Understanding disabilities

\section{Introduction}

The social and physical environments surrounding people with disabilities have been modified, in terms of sociology, economics, and politics, to create an inclusive society. For example, many countries have changed or established laws relating to disability policy and the maintained living environment of people with disabilities. However, a society may still not be regarded as inclusive (especially in Japan), even if social and physical environments are modified, if the public continue to hold emotional stereotypes toward people with disabilities. Thus, we also need to focus on psychological variables, such as impressions and attitudes toward people with disabilities, to minimize such stereotype.

In order to promote this trend, it is necessary for the public to acquire an understanding of disabilities. Specifically, we should investigate background factors affecting the formation of impressions and attitudes toward people with disabilities in the public. Many previous studies have provided beneficial findings using various methodologies, such as the Picture-Ranking method (Richardson, 1971; Richardson, Goodman, Hasdorf, \& Dornbusch, 1961; Richardson \& Royce, 1968), the Sociometric method (Begab, 1970), and the Interview method (Conant \& Budoff, 1983). Questionnaire methods also have been adopted in many previous studies, such as the ATDP (Attitudes Toward Disabled Persons Scale: Yuker, Block, \& Young, 1986) and the SD (semantic differential) method (Ahlborn et al., 2008; Greenbaum \& Wang, 1965; Panek \& Jungers, 2008; Panek \& Smith, 2005; Takahashi \& Haga, 2016). Specifically, the SD method has been adopted in many previous studies to examine affective responses toward people with disabilities.

The SD method, developed by Osgood, Suci, and Tannenbaum (1957), has been widely used for measuring affectively related variables underlying impressions of various stimuli. The established SD technique requires participants to rate affective responses to stimuli on bipolar scales defined by contrasting adjective pairs, e.g., "likable-dislikable." Then, the factors underlying the multivariate data are extracted by conducting a factor analysis to explain the affective responses in terms of a limited number of specific main factors. Osgood et al. (1957) proposed that three semantic categories are generally extracted across various materials and different cultures: Evaluation, Activity, and Potency. 
Since it is easy to calculate scores in the SD method, researchers can examine impressions quantitatively (Heise, 1970). Under this view, the SD method has proved useful in the study of impressions toward disabilities, because it provides a direct experimental method to measure affective impressions toward disability-related concepts (Antonak \& Linvneh, 1991). Regarding the SD method, previous studies have provided evidence that the abovementioned three factors underlie in the formation of affective impressions toward disabilities, focusing on intellectual disability (Ahlborn, Panek, \& Jungers, 2008; Dhillon \& Chaudhuri, 1990; Jaffe, 1966; Nosse \& Gavin, 1991; Panek \& Jungers, 2008; Panek \& Smith, 2005; Rees Spreen, \& Harnadek, 1991; and see also the review by Scior, 2011) and speech disorder (Lass, Ruscello, Bradshaw, \& Blankenship, 1991; Lass, Ruscello, Stout, \& Hoffman, 1991; Ma \& Yu, 2013; Ruscello, Lass, \& Odbesek, 1988). For example, Ahlborn et al. (2008), using the SD method, experimentally manipulated gender (male/female) and age (3, 12, 20 years) of a person with an intellectual disability and measured impressions. They revealed that impressions of people with intellectual disabilities became more negative with increasing age in Activity, but not in Evaluation or Potency. Panek and Jungers (2008) also used the SD method and examined the effects of age, gender, and cause of disability on impressions of people with intellectual disabilities. Results showed that intellectual disability due to genetic factors (e.g., Down syndrome) was evaluated more positively than self-inflicted intellectual disability (e.g., brain damage due to drinking cleaning fluid) in Evaluation, but not in Activity or Potency. They also showed sex differences, in that female participants positively rated on the SD scale compared with male participants in the Evaluation and Potency.

Almost all previous studies using the SD method focused mainly on intellectual disability. For example, Ahlborn et al. (2008) studied the term intellectual disability; Panek and Smith (2005) studied the terms mentally challenged, intellectual disability, cognitive disability, cognitive-adaptive disability, developmental disability, mentally retarded, visually disabled, and physically disabled; Panek and Jungers (2008) studied the term intellectual disability; Greenbaum and Wang (1965) studied the terms mentally retarded and mentally ill (mentally ill, emotionally disturbed, and neurotic); and Takahashi and Haga (2016) examined intellectual and developmental disabilities. Regarding the categorization of disabilities, previous studies proposed the selection of disabilities as experimental stimuli in questionnaire-type studies according to whether they are physical or mental, and whether they are observable or not (e.g., Furnham \& Pendred, 1983). For example, using the questionnaire method of ATDP, Furnham and Pendred (1983) adopted blindness (physical/observable), deafness (physical/nonobservable), Down's syndrome (mental/observable), and educationally subnormal (mental/non-observable) as stimuli, and showed that attitudes toward physically disabilities were more positively rated than those toward mental disabilities, whereas there were no differences between observable and non-observable disabilities. Furnham and Gibbs (1984) also used the ATDP and observed more positive evaluations of physical disabilities compared with mental disabilities in schoolchildren. Including these results, previous studies have generally demonstrated that the public rate physical disability positively compared with mental disability. However, these findings have mainly arisen from use of the ATDP, and to the best of my knowledge, there are very few findings using the SD method. One previous study (Panek \& Smith, 2005) examined differences in impressions among intellectual disability (e.g., mentally challenged and mentally retarded), visual disability, and physical disability using the SD method. Their results showed that the term mentally challenged was more positive than the term visually disabled, and no other differences were observed. While the authors hypothesized that terms associated with intellectual disability (e.g., mentally challenged and mentally retarded) would be negatively rated compared with the terms visually disabled and physically disabled based on previous findings of positive ratings for physical disability, their results did not show clear differences in impressions among these disabilities.

The aim of the present study was to measure the affective impressions of various disabilities, focusing on the terms intellectual disability, physically handicapped, weakness, blindness, deafness, and developmental disability and to examine differences in impressions between these disabilities, using the SD method. Considering the positive ratings for physical disability compared with mental disability obtained by various studies, I predicted that physical disabilities (physically handicapped, weakness, blindness, and deafness) would be rated positively compared with mental disabilities (intellectual disability and developmental disability) in any of the factors extracted by the SD method. On the other hand, if no differences in impressions among these disabilities could be observed, as Panek and Smith (2005) found, this would show a methodological distinction in which positive ratings for physical disability compared with mental disability may be observed when using the ATDP, but not using the SD method. 


\section{Material and Methods}

\section{1. Participants}

Participants in the SD experiment were 50 university students (18 men and 32 women; mean age $=20.52$, SD $=1.28$ ). They had received token lessons in the special needs education required to become a teacher in a special needs education school. All participants provided informed consent before participating.

\section{2. Stimuli}

Participants rated their impressions of six terms relating to disabilities: intellectual disability, physically handicapped, weakness, blindness, deafness, and developmental disability. The present study adopted these terms as stimuli because these disabilities are the main targets of the special needs education system in Japan.

\subsection{Adjective pairs}

Twenty adjective pairs (see Table 1) were selected on the basis of previous studies examining impressions toward disabilities (Ahlborn et al., 2008; Ma \& Yu, 2013; Panek \& Smith, 2005; Takahashi \& Haga, 2016). In the analysis, four adjective scales ("sharp-blunt," "stable-unstable," "clear-cloudy," and "wet-dry") were removed, as described in the results section.

\section{4. Procedure}

Each participant rated at his/her own pace the disability-related terms, which were presented one-by-one printed on paper, on six-point scales relating to 20 adjective pairs (e.g., $1=$ dynamic to $6=$ static). All participants rated six disability-related terms, and the presentation order of the terms was counterbalanced across participants. Time required for the SD experiment was about 15 minutes.

\section{Results}

\section{1. Factor analysis results}

I conducted a factor analysis on the combined data from ratings of six disability-related terms by 50 participants ( 300 data points $=50$ participants $\times 6$ terms). Although I conducted the factor analysis (using the principal factor method) with promax rotation, low initial values $(<0.20)$ for two adjective pairs ("sharp-blunt" and "wet-dry") and low factor loadings $(<0.30)$ for two adjective pairs ("stable-unstable" and "clear-cloudy") were observed. I removed these four adjective pairs and again conducted factor analysis with promax rotation and extracted three factors. Table 1 shows the factor loadings after promax rotation. The first factor accounted for $33.91 \%$ of the total variance, and included the "dynamic-static," "gay-sober," and "lively-quiet" scales. The second factor accounted for $17.55 \%$ of the total variance, and included the "likable-dislikable," "pleasant-unpleasant," and "beautifulugly" scales. The third factor accounted for $9.37 \%$ of the total variance, and included the "soft-hard," "smoothrough," and "light-heavy" scales. These factors could be regarded as Activity, Evaluation, and Potency, respectively, according to the terminology of Osgood et al. (1957) and considering previous studies (e.g., Takahashi \& Haga, 2016). Furthermore, I calculated Cronbach's $\alpha$ for all scales to confirm the reliability of each factor. The $\alpha$-value for the first factor (Activity) was 0.89, for the second factor (Evaluation) was 0.82, and for the third factor (Potency) was 0.68 . 
Table 1. Factor loadings after promax rotation

\begin{tabular}{l|lll}
\hline item & $\begin{array}{l}\text { Factor I } \\
\text { (Activity) }\end{array}$ & $\begin{array}{l}\text { Factor II } \\
\text { (Evaluation) }\end{array}$ & $\begin{array}{l}\text { Factor III } \\
\text { (Potency) }\end{array}$ \\
\hline dynamic-static & .838 & .029 & -.053 \\
gay-sober & .800 & -.015 & -.030 \\
lively-quiet & .778 & -.116 & .109 \\
excited-calm & .774 & -.207 & -.149 \\
active-passive & .758 & .189 & .036 \\
cheerful-gloomy & .711 & .179 & .119 \\
powerful-feeble & .663 & -.003 & -.014 \\
strong-weak & .518 & .187 & .032 \\
rugged-delicate & .456 & -.390 & .055 \\
likable-dislikable & .066 & .861 & -.073 \\
pleasant-unpleasant & .028 & .812 & .013 \\
beautiful-ugly & -.054 & .672 & .040 \\
soft-hard & -.006 & -.070 & .771 \\
smooth-rough & -.134 & .069 & .706 \\
light-heavy & .141 & -.049 & .479 \\
relaxed-tense & .057 & .004 & .454 \\
\hline Eigen value & 5.43 & 2.81 & 1.50 \\
Contribution of each factor $(\%)$ & 33.91 & 17.55 & 9.37 \\
Cumulative contribution $(\%)$ & 33.91 & 51.46 & 60.83 \\
\hline
\end{tabular}

Note: Cronbach's $\alpha$ for scales based on Factor I (Activity) $=0.89$, Factor II (Evaluation) $=0.82$, and Factor III $($ Potency $)=0.68$. Although the present study used 20 adjective pairs in the SD experiment, I removed "sharpblunt" and "wet-dry" because of low initial value and "stable-unstable" and "clear-cloudy" because of low factor loadings in the factor analysis.

\section{2. Differences in factor scores between six disability-related terms}

In order to examine differences in factor scores between the six disability-related terms, I conducted a one-way repeated measures ANOVA in each extracted factor, with disability (6; intellectual disability, physically handicapped, weakness, blindness, deafness, and developmental disability) as a within-participants factors (Figure $1)$.

For the Activity factor scores, the results showed a main effect of disability $\left[F(5,245)=51.19, p<0.001, \eta_{p}{ }^{2}=\right.$ 0.51]. Post-hoc analysis (Tukey's HSD method) revealed that scores on this factor were higher for physically handicapped, weakness, blindness, and deafness than for intellectual disability and developmental disability [physically handicapped vs. intellectual disability: $t(245)=9.54, p<0.05$; physically handicapped vs. developmental disability: $t(245)=9.55, p<0.05$; weakness vs. intellectual disability: $t(245)=11.38, p<0.05$; weakness vs. developmental disability: $t(245)=11.39, p<0.05$; blindness vs. intellectual disability: $t(245)=$ 9.05, $p<0.05$; blindness vs. developmental disability: $t(245)=9.06, p<0.05$; deafness vs. intellectual disability: $t(245)=8.47, p<0.05$; deafness vs. developmental disability: $t(245)=8.48, p<0.05]$. Moreover, the factor score for weakness was higher than the score for deafness $[t(245)=2.91, p<0.05]$.

For scores on the Evaluation factor, the results showed a main effect of disability $[F(5,245)=10.28, p<0.001$, $\left.\eta_{p}{ }^{2}=0.17\right]$. Post-hoc analysis (Tukey's HSD method) revealed that scores on this factor were higher for intellectual disability, physically handicapped, weakness, and developmental disability than for blindness and deafness [intellectual disability vs. blindness: $t(245)=3.14, p<0.05$; intellectual disability vs. deafness: $t(245)=$ $3.42, p<0.05$; physically handicapped vs. blindness: $t(245)=4.53, p<0.05$; physically handicapped vs. deafness: $t(245)=4.81, p<0.05$; weakness vs. blindness: $t(245)=4.59, p<0.05$; weakness vs. deafness: $t(245)=$ 4.87, $p<0.05$; developmental disability vs. blindness: $t(245)=4.25, p<0.05$; developmental disability vs. deafness: $t(245)=4.53, p<0.05$ ] 
For scores on the Potency factor, the results showed a main effect of disability $\left[F(5,245)=8.57, p<0.001, \eta_{p}{ }^{2}=\right.$ 0.15]. Post-hoc analysis (Tukey's HSD method) revealed that factor scores for physically handicapped were higher than scores for other disabilities [physically handicapped vs. intellectual disability: $t(245)=5.32, p<0.05$; physically handicapped vs. weakness: $t(245)=2.82, p<0.05$; physically handicapped vs. blindness: $t(245)=$ 4.87, $p<0.05$; physically handicapped vs. deafness: $t(245)=5.13, p<0.05$; physically handicapped vs. developmental disability: $t(245)=4.70, p<0.05$ ].

\subsection{Differences in factor scores between male and female participants}

In order to reveal sex differences in factor scores between male and female participants in relation to six disability-related terms, I conducted a two-way repeated measures ANOVA in each factor, with sex (2; male and female) as a between-participants factor and disability (6; intellectual disability, physically handicapped, weakness, blindness, deafness, and developmental disability) as a within-participants factor. Sex differences were observed only in Activity: for this factor, a significant interaction between sex and disability was observed $[F(5$, 240) $\left.=3.29, p<0.01, \eta_{p}{ }^{2}=0.06\right]$, showing that male participants gave more negative ratings compared with female participants for deafness $\left[F(1,288)=5.86, p<0.05, \eta_{p}{ }^{2}=0.02\right]$. However, I did not observe sex differences in Evaluation [main effect of sex: $F(1,48)=0.11, p=0.74, \eta_{p}{ }^{2}=0.002$; interaction between sex and disability: $F(5,240)=1.30, p=0.26, \eta_{p}{ }^{2}=0.03$ ] or in Potency [main effect of sex: $F(1,48)=0.03, p=0.96, \eta_{p}{ }^{2}=$ 0.0006 ; interaction between sex and disabilities: $F(5,240)=0.45, p=0.81, \eta_{p}{ }^{2}=0.009$ ].

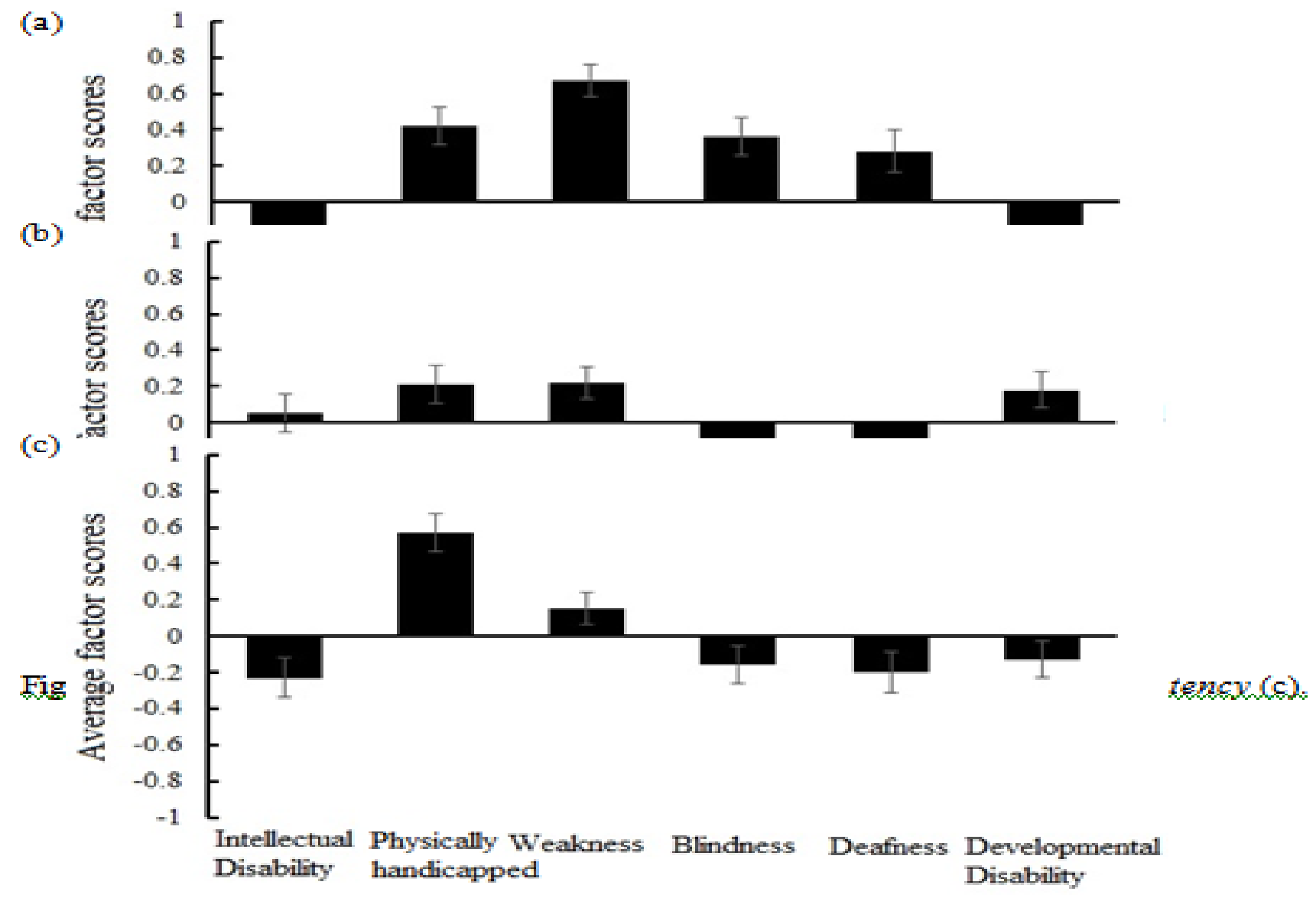

\section{Discussion}

This study measured affective impressions of various disabilities using the SD method and compared them among disabilities. In the SD experiment, participants rated six disability-related terms (intellectual disability, physically handicapped, weakness, blindness, deafness, and developmental disability) on 20 adjective-pair scales. I conducted a factor analysis to extract factors relating to impressions toward disabilities, and compared factor scores among disabilities to reveal differences in impressions. 
In the factor analysis, I extracted three factors (Activity, Evaluation, and Potency). In the SD method, as Osgood et al. (1957) indicated, three factors are generally extracted, even if the researcher uses varying experimental stimuli in different cultures. The present study confirmed the results of Takahashi and Haga (2016) in which the authors also proposed three factors underlying impressions toward disabilities. Although the types of disability used in the present study differed from previous studies (e.g., Ahlborn et al., 2008; Greenbaum \& Wang, 1965; Panek \& Jungers, 2008; Panek \& Smith, 2005; Takahashi \& Haga, 2016), similar factors were assumed to be extracted using the SD method, and these three factors were assumed to affect the formation of impressions toward disabilities.

On the basis of these factor scores, analysis revealed differences in affective impressions toward various disabilities. In Activity, factor scores for physically handicapped, weakness, blindness, and deafness were higher than those for intellectual disability and developmental disability. This factor included adjective pairs such as the "dynamic-static," "gay-sober," and "lively-quiet" scales, and high factor scores represented negative valence (i.e., static, sober, and quiet). Thus, I inferred that physically handicapped, weakness, blindness, and deafness were rated more static, sober, and quiet compared with intellectual disability and developmental disability. In Evaluation, factor scores for intellectual disability, physically handicapped, weakness, and developmental disability were higher than those for blindness and deafness. This factor included adjective pairs such as the "likable-dislikable," "pleasant-unpleasant," and "beautiful-ugly" scales, and higher factor scores represented negative valence (i.e., dislikable, unpleasant, and ugly). Thus, I inferred that intellectual disability, physically handicapped, weakness, and developmental disability were rated more dislikable, unpleasant, and ugly compared with blindness and deafness.

In Potency, factor scores for physically handicapped were higher than those for other disabilities. This factor included adjective pairs such as the "soft-hard," "smooth-rough," and "light-heavy" scales, and high factor scores represented negative valence (i.e., hard, rough, and heavy). Thus, I inferred that physically handicapped was rated harder, rougher, and heavier.

The main purpose of the present study was to examine differences in impressions among various disabilities using the SD method, in relation to disabilities categorized as both physical and mental. I predicted that physical disabilities (physically handicapped, weakness, blindness, and deafness) may be rated positively compared with mental disabilities (intellectual disability and developmental disability) on any factor. Although the present results has described variation in impressions among disabilities that differs from the results of Panek and Smith (2005), also using the SD method, the present results additionally did not support the results of Furnham and Pendred (1983), who used the ATDP; physical disabilities (physically handicapped, weakness, blindness, and deafness) were rated negatively and mental disabilities (intellectual disability and developmental disability) were rated positively in Activity, and in Evaluation and Potency, there were no clear tendencies toward a difference between the physical and mental categories. Thus, I suggest that the SD method, as distinct from the other questionnaire method of the ATDP, might provide an alternative perspective for examining impressions toward disabilities.

I propose that these ambiguous results could be understood by considering the sensory/perceptual modalities underlying each factor. Previous studies (Suzuki \& Gyoba, 2001, 2003; Suzuki Gyoba, Kawabata, Yamaguchi, \& Komatsu, 2006) proposed a relationship between the adjective scales that make up the three factors of the SD method and sensory/perceptual-relevance properties. They examined the relationship between sensory modalities (i.e., cold, warm, gustatory, olfactory, tactual, kinesthetic, equilibrium, pain, auditory, and visual) and 75 adjective scales that have been used in the SD method. Results showed that the adjective pairs included in Activity and Potency were associated with high relevance magnitudes for the auditory/kinesthetic and tactile modalities, respectively, indicating that these can be regarded as specific modality scales. The adjective pairs included in Evaluation were generally associated with high relevance magnitudes for multiple modalities, indicating that this factor can be regarded as a multi-modality scale. These behavioral findings are almost consistent with results from the brain activity using twenty-four channels near-infrared spectroscopy (NIRS) (Suzuki, Gyoba, \& Sakuta, 2003, 2005). According to these findings, adjective pairs belonging to Activity and Potency may be associated with the auditory/kinesthetic modalities and the tactile modality, respectively. Moreover, Evaluation may be associated with multi-modality scales. On the basis of these sensory/perceptual properties underlying each factor, may be surmised that physically handicapped, weakness, blindness, and deafness might be rated negatively in Activity because these disabilities relate to kinesthetic scales and this may be a major factor affecting the rating. 
In Evaluation, intellectual disability, physically handicapped, weakness, and developmental disability might be rated negatively because these disabilities relate to multi-modality scales and again this may be an important factor in the rating. In Potency, since physically handicapped relates to the tactile modality, it might be rated negatively for this reason. I speculate that the sensory/perceptual properties pertaining to each factor might affect impressions of disabilities. In addition to the categorization of a disability as physical or mental, as previous studies have indicated (e.g., Furnham \& Pendred, 1983), there might be a possibility that sensory/perceptual properties affect the formation of impressions toward disabilities.

The present study has several limitations. First, although I have discussed the present results in terms of the sensory/perceptual analysis underlying each factor, future research should reveal this empirically. For example, Suzuki and Gyoba (2003) and Suzuki et al. (2006) measured the sensory-relevance properties of each adjective scale and examined the relationship between sensory-relevance properties and three factors. By conducting this type of evaluation task using various disabilities as experimental stimuli, the relationship between sensory/perceptual properties of adjective scales within each factor and impressions toward disabilities could be empirically examined, which may support my claim for the present results. Second, wider variety of disabilities should be examined to reveal differences in impressions of them although the present study focused on the main targets of the special needs education system in Japan. Third, future research should explore the factors that relate to more positive impressions. Generally, factors such as experiences of contact and degree of recognition of a particular disability affect the formation of impressions and attitudes toward people with disabilities. Examining the effects of these factors on the impressions of various disabilities extracted by this study may provide a method of promoting positive impressions toward people with disabilities. This work may contribute ideas for enhancing public understanding of disabilities and decreasing prejudice and discrimination towards people with disabilities.

\section{Conclusions}

The present study extracted three factors (Activity, Evaluation, and Potency) in a factor analysis by conducting an SD experiment in which participants rated disability-related terms (intellectual disability, physically handicapped, weakness, blindness, deafness, and developmental disability) on various adjective scales. On the basis of each factor score, analysis revealed significant negative impressions on the Activity factor for physically handicapped, weakness, blindness, and deafness compared with intellectual disability and developmental disability; on the Evaluation factor for intellectual disability, physically handicapped, weakness, and developmental disability compared with blindness and deafness; and on the Potency factor for physically handicapped compared with other disabilities. Regarding the sensory/perceptual analysis proposed by previous studies (namely, that Activity, Evaluation, and Potency relate to auditory/kinesthetic modalities, multi-modalities, and the tactile modality, respectively), the present results might suggest that physically handicapped, weakness, blindness, and deafness were negatively rated in Activity because they relate to the kinesthetic modality; that intellectual disability, physically handicapped, weakness, and developmental disability were negatively rated in Evaluation because they relate to multi-modalities; and that physically handicapped was negatively rated in Potency because it relates to the tactile modality. These results improve understanding of how affective impressions towards disabilities vary depending on the type of disability in relation to the sensory/perceptual properties underlying each factor.

\section{Acknowledgements:}

This work was supported by a JSPS (Japan Society for the Promotion of Science) Research Fellowship to J. T. (Grant No. 17K17616).

\section{References}

Ahlborn, L. J., Panek, P. E., Jungers, M. K. (2008). College students' perceptions of persons with intellectual disability at three different ages. Research in Developmental Disabilities, 29, 61-69.

Antonak, R. F., Livneh, H. (1991). Survey research on attitudes. Matson, J. L. \& Mulick, J. A. (Eds.), Handbook of mental retardation ( $2^{\text {nd }}$ ed., pp. 552-568). New York: Pergamon Press.

Begab, M. J. (1970). Impact of educational in social work student's knowledge and attitudes about mental retardation. American Journal of Mental Deficiency, 94, 801-808.

Conant S., Budoff M. (1983). Patterns of awareness in children's understanding of disabilities. Mental Retardation, 21, 119-125. 
Dhillon, P. K., Chaudhuri, S. (1990). A study of the "meaning" of the concept of mental retardation as held by the community. Manas, 37, 1-8.

Furnham, A., Gibbs, M. (1984). School children's attitudes towards the handicapped. Journal of Adolescence, 7, 99117.

Furnham, A., Pendred, J. (1983). Attitudes towards the mentally and physically disabled. British Journal of Medical Psychology, 56, 179-187.

Greenbaum, J. J., Wang, D. D. (1965). A semantic-differential study of the concepts of mental retardation. The Journal of General Psychology, 73, 257-272.

Heise, D. R. (1970). The semantic differential in attitude research. Summers, G. F. (Ed.), Attitude measurement. Chicago, IL: Rand McNally.

Jaffe, J. (1966). Attitudes of adolescents toward the mentally retarded. American Journal of Mental Deficiency, 70, 907-912.

Lass, N. J., Ruscello, D. M., Bradshaw, K. H., Blankenship, B. L. (1991). Adolescents' perceptions of normal and voice-disordered children. Journal of Communication Disorders, 24, 267-274.

Lass, N. J., Ruscello, D. M., Stout, L. L., Hoffman, F. M. (1991). Peer perceptions of normal and voice-disordered children. Folia Phoniatrica et Logopaedica, 43, 29-35.

Ma, E. P.-M., Yu, C. H.-Y. (2013). Listeners' attitudes toward children with voice problems. Journal of Speech, Language, and Hearing Research, 56, 1409-14115.

Nosse, L. J., Gavin, K. J. (1991). Influence of direct contact on college students' attitude toward adults with mental handicaps. College Student Journal, 25, 201-206.

Osgood, C. E., Suci, G. J., Tannenbaum, P. H. (1957). The measurement of meaning. Urbana: University of Illinois Press.

Panek, P. E., Jungers, M. K. (2008). Effects of age, gender, and causality on perceptions of persons with mental retardation. Research in Developmental Disabilities, 29, 125-132.

Panek, P. E., Smith, J. L. (2005). Assessment of terms to describe mental retardation. Research in Developmental Disabilities, 26, 565-576.

Rees, L. M., Spreen, O., Harnadek, M. (1991). Do attitudes towards persons with handicaps really shift over time? Comparison between 1975 and 1988. Mental Retardation, 29, 81-86.

Richardson, S. A. (1971). Children's values and friendship: A study of physical disability. Journal of Health and Social Behavior, 12, 253-258.

Richardson, S. A., Goodman N., Hasdorf A., Dornbusch S. (1961). Cultual uniformity in reaction to physical disabilities. American Sociological Review, 26, 241-247.

Richardson, S. A., Royce J. (1968). Race and physical handicap in children's preference for other children. Child Development, 39, 467-480.

Ruscello, D. M., Lass, N. J., Podbesek, J. (1988). Listeners' perception of normal and voice-disordered children. Folia Phoniatrica et Logopaedica, 40, 290-296.

Scior, K. (2011). Public awareness, attitudes and beliefs regarding intellectual disability: A systematic review. Research in Developmental Disabilities, 32, 2164-2182.

Suzuki, M., Gyoba, J. (2001). Contrastive analysis of sensory-relevance of factors affecting aesthetic impressions. Tohoku Psychologica Folia, 60, 81-92.

Suzuki, M., Gyoba, J. (2001). Analyzing the factor structure and the sensory-relevance of impressions produced by words and drawings. The Japanese Journal of Psychology, 73, 518-523. [in Japanese with English abstract]

Suzuki, M. Gyoba, J., Kawabata, H., Yamaguchi, H., Komatsu, H. (2006). Analysis of the sensory-relevance of adjective pairs by the modality differential method. The Japanese Journal of Psychology, 77, 464-470. [in Japanese with English abstract]

Suzuki, M., Gyoba, J., Sakuta, Y. (2003). Multichannel near-infrared spectroscopy analysis of brain activities during semantic differential rating of drawings. Tohoku Psychologica Folia, 62, 86-98.

Suzuki, M., Gyoba, J., Sakuta, Y. (2005). Multichannel NIRS analysis of brain activity during semantic differential rating of drawing stimuli containing different affective polarities. Neuroscience Letters, 375, 53-58.

Takahashi, J., Haga, A. (2016). Evaluation of impressions toward people with intellectual and developmental disabilities using semantic differential method. International Journal of Humanities and Social Science, 6, 257262.

Yuker, H. E., Block, J. R., Young, J. H. (1986). Research with the attitudes towards disabled persons scales (ATDP), Hempstead. New York: Hofstra University Press. 